\title{
Neuromorphic Computing - The Principal of Development
}

\author{
R. Sathya Bama Krishna ${ }^{1}$, D. Usha Nandini' ${ }^{2}$ J. Albert Mayan ${ }^{3}$, \\ Nehal Veeramachaneni ${ }^{4}$, Nidhi Sawarn ${ }^{5}$ \\ \{ $\underline{\text { rsathyarajeswari@gmail.com }}^{1}$, usha.cse@ sathyabama.ac.in ${ }^{2}$, albertmayan@gmail.com ${ }^{3}$ \\ vn001030@gmail.com ${ }^{4}$, nidhisawarnn29@gmail.com $\left.{ }^{5}\right\}$
}

\begin{abstract}
1,2 Assistant Professor, Department of Computer Science and Engineering, Sathyabama Institute of Science and Technology, Chennai 600119, India, ${ }^{3}$ Associate Professor, Department of Computer Science and Engineering, Sathyabama Institute of Science and Technology, Chennai 600119, India,

${ }^{4,5}$ Student, Department of Computer Science and Engineering, Sathyabama Institute of Science and Technology, Chennai 600119, India
\end{abstract}

\begin{abstract}
Due to the subsequent decline of Moore's Law, everyone is in the search for an approach to push computing technology much further. Neuromorphic Computing is a way through which we can approach the next generation of computer technology. Neuromorphic chips have been the current trending topic as it deals with a diverse range of tactics to formulate the given information to not just make a particular decision but it also modifies the network to memorize the information and finish the work more efficiently. The progress in VLSI mechanics through time and the further research and development on neuromorphic computing lead to a stable growth in the ability and competence of neuromorphic engineering in applications. These chips perform complex tasks such as image recognition, navigation and problem solving. In this paper we present the history, the need for Neuromorphic computing, the functionalities, the current projects, their main features and technical capabilities of Neuromorphic computing.
\end{abstract}

Keywords: neuro-inspired model, neuromorphic computing, artificial intelligence, computational modelling.

\section{Introduction}

Neuromorphic computing or engineering is a subset of artificial intelligence in which scientists develop devices which work on the principles of the human brain. It is more like making devices which mimics the function of the human brain. The computing of neuromorphic devices involves the development of components which are analogous to the human brain. Talking about the structure of these devices, these are definitely not in the shape of the brain; however they do fulfil the roles of their organic counterparts. To sum it up, neuromorphic computing involves making inorganic brains.

Traditional computers or digital computers work in binary. It understands and responds in either $0 \mathrm{~s}$ or $1 \mathrm{~s}$ or yes or no. This type of mechanism is very narrow in the field of developing science. Therefore, the way to solve problems in digital computers is structured in a very rigid way. These mechanisms cannot move beyond the binary language. On the other hand, neuromorphic computers are very flexible and their approach to solving problems is very 
broad. One of the ways to move past this threshold is by making computers more like human brains. Neuromorphic computing is a concept in which the computer chips are designed in a way which uses the science of computation based on the human nervous system. Neuromorphic computers are more energy efficient than the digital computers. It is designed so that the neurons can learn as they perform tasks. The hope behind this new revolutionary discovery is to make a computer that behaves like a brain which will give us enough computing power to simulate something as complicated as the brain.

\section{Neuromorphic Computing}

One of the most powerful computers out there in the world is a human brain. It consists of 100 billion neurons while each neuron is having 100-1000 of synapses. A neuron (cell which transmits information to other parts of the body from the brain). Synapses are the connection to other neurons. A human brain can perform 1 billion calculations per second. Many researches are being done to make supercomputers which can reach this exa-scale performance. Simulating a human brain requires millions of processors and high-speed memory using power consumption in the order of megawatts per hour. While a brain can do all of this with just 20 watts of power consumption and will still outperform these supercomputers.

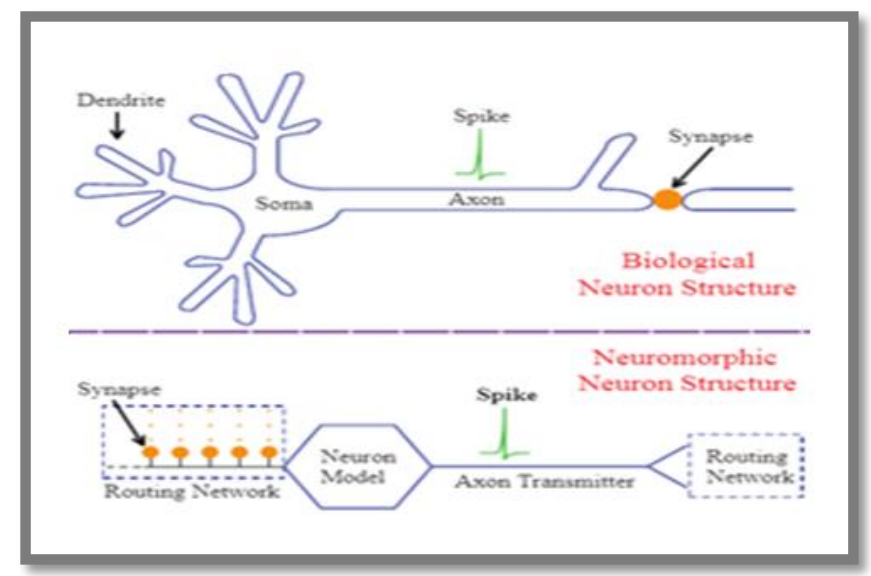

Fig.2.1 Simplified biological and neuromorphic neuron structure

All of this is due to the differentiation of modern computer architectures and a biologically cognitive brain. A modern computer's architecture is based on von Neuman architecture, in which memory and computation is isolated with a data bus connecting them. Whereas a biological brain has memory and computation tightly coupled together. The von Neuman architecture is the best architecture for digital computers. But to simulate the brain and make computers work the way the brain works, we need a different kind of structure more similar to the human brain,

Neuromorphic architecture where the Spinal neural network is the population of neurons connected to other neurons by the help of synapses. Synapses are the junction between neurons. This network connection is responsible for the transmission of information to 
different parts of body. Neurons have axons, a protoplasmic protrusion (in simpler words a joint) which is extended to different parts of body.

These connections between neurons are much more complex than artificial neural network (ANN). Synapses are of two types.

- Chemical synapses

- Electrical synapses

Some examples of neural circuits are

1. Trisynaptic circuit in the hippocampus

2. Papez circuit (links hypothalamus to limbic lobe)

3. Central pattern generators in spinal cord.

The main part of this whole neuromorphic computing is the artificial neural networks (ANN). These neural networks are framed with artificial neurons which are microscopic computational units designed to carry out simple mathematical functions. Our brain is composed of neurons. Neurons are the cells which transfer the information to other parts of the body from the brain. But the Artificial Neurons is not of much use alone. To use them efficiently, they need to be stacked up in layers to perform complex tasks. For example, object detection within given images, converting voice and audio as to texts. Recently, some of the artificial intelligence researchers exhibited a self-driving bicycle that navigates around and detects obstacles, follows a particular person and also responds to his voice commands. To manufacture bicycles of such advancement, a neuromorphic chip was used.

Traditional computing system relies on processing units which poses a lot of power and could perform complex tasks with high speed, but it is tough to run neural networks on these computers. However, processing units designed for Graphics, hardware's employed for games mostly perform a lot of parallel processing jobs. Matrix multiplication, a core application with neural networks can also be done using GPUs. GPU arrays play a vital role in most neural network applications. Neuromorphic computers run AI models role over much better than CPUs and GPUs with less power consumption.

Digital computers work on the principle of determinism. The work of a digital computer is to perform a task or solve a problem based on some inputs given by users. Its work is predetermined. Whereas, neuromorphic computers are designed to think and process, to learn from the applications performed just like human brain works. Plasticity of neurons makes it the more suitable feature of nervous systems to modify its activity in response to intrinsic or extrinsic stimuli thereby transforming its functions, structure, or connections. This is one of the phenomena, neuromorphic engineers are hoping to simulate.

\section{The Saga of Neuromorphic Computing}

Neuromorphic computing, a vivacious concept which was evolved by Carver Mead during late 1980s in which he employed a very large-scale integration system with electric analog circuits that mimic the functional portions of the nervous system.

The idea of neuromorphic computing was first given by Turing a scientist who formally proved that a machine can perform any complex mathematical computation if it is represented 
as an algorithm. He wrote a book called Intelligent Machinery which was published in 1958. In his book, he described a machine consisting of artificial neurons arranged in any pattern with modifier devices. He explained those modifiers could be configured to pass or destroy a signal and the neurons were composed of NAND gates.

Later on, Synaptic plasticity came into picture. It is basically the ability of synapses to strengthen and weaken themselves over time in order to increase or decrease their activity, basically, a mechanism for learning and memory. It was given by Hebb in 1949 .

In 1980, VLSI pioneer Mead along with Conway published the landmark text introduction to VLSI systems. Later, he teamed up with John Hopfield and Feynman to study how animal brains compute. This study catalyzed the field of neural networks (Hopfield), neuromorphic engineering (Mead) and physics of computation (Feynman).

Mead created the world's first neural-inspired chips including an artificial retina and cochlea, which was documented in his book Analog VLSI Implementation of Neural Systems published in 1989.

In the year 2008, HP Laboratories had set forth the production of postulated electronic equipment of Chua's, the memristor and stated their usages as synapses within neuromorphic circuits.

\section{A. THE NEED}

Moore's law defines that every two years, quantity of transistors within a microchip doubles, with the cost of computer being halved. In 1965, Gordon \&amp; Moore, the cofounder of intel, made this observation that became Moore's law.

In today's scenario, the way we are moving forward and the excessive demand of memory chips being smaller and smaller in size, it will be practically impossible to store so much of data into smaller chips. As Moore's law is nearly towards the end, silicon processing technology is being pushed towards its limits. But we can't continue with decreasing the size of chips for better computational powers. Volleytronic transistors are being developed having size in nanoscale. They have proved to be promising to save the growth potential of this technology. But many other developments are being done to save Moore's law.

Neuromorphic computing is one of the many developments being made in this technology. Neuromorphic computing aims to make computers better at operating. It would allow computers to adapt and learn faster, this makes the use of programming to be reduced accordingly. The architecture of neuromorphic computers and its ability to learn makes it work more efficiently at running neural networks. The structure is designed in such a way that the Artificial Intelligence versions runs with much faster speed than the central processing units and GPUs while using less amount of energy. Which is an important aspect since Artificial Intelligence gives a concern for the efficient usage of energy.

Neuromorphic computers use less energy, are small in size and it can process data and take decisions on its own without the need to depend upon a certain connection. This technology in the Global Sector could mean gigantic development and upgrade of industries in various environments. This could accelerate the progress of mankind in development of robotics and autonomous technology.

\section{B. NEUROMORPHIC CHIPS THE MOVEMENT OF AI}

The life span to traditional programming-to manually formulates or code rules are coming to an end. Moore's law says that the number of transistors in a dense integrated circuit double 
for every two years has come to a break down point. The transistors currently in market are about 70 silicon atoms wide, therefore the chances of making them even smaller are diminishing. So, it is needed for us to change the approach by taking a step further by improving the abilities that a computer can do.

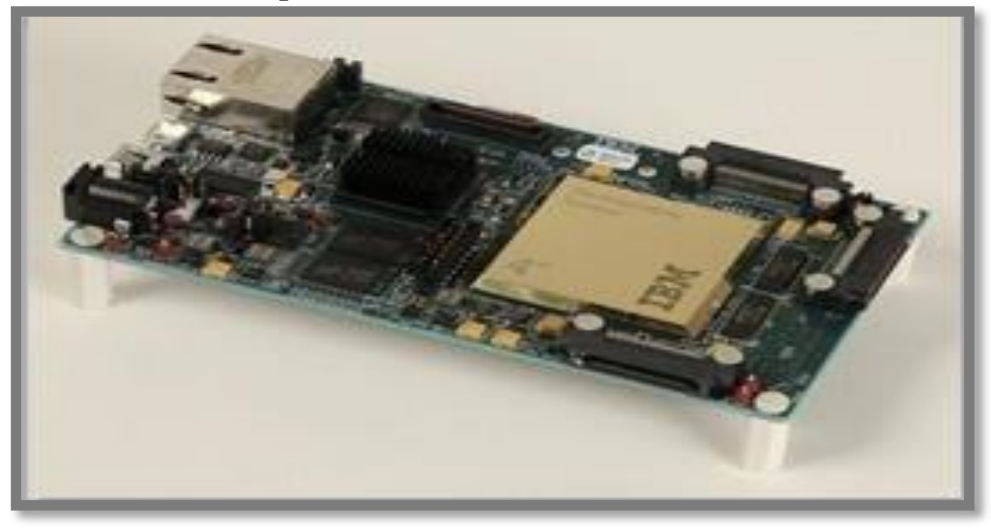

Fig.3.1 Neurosynaptic Chip

A neuromorphic chip is an analog processor chip whose functionalities are being designed to operate like a human brain. It resembles the natural organic structures of our nervous system by enacting neurons in silicon. In the brain the neurons and their connections are synapses. The neuromorphic chips are arranged in such a way that they act like artificial neural networks. Each neuromorphic chip has a tiny computation unit that represents an artificial neuron. An artificial neuron network has a layered structure and each network node has the ability to process input and send output to other nodes. This artificial neural network contributes the neuromorphic chip to act like a brain.

Artificial Neural Networks have the ability to handle and analyze nonlinear and complex data therefore generalizing and predicting data. The network structure has an input layer, hidden layer and output layer. Due to the presence of multi layers it is also known as Multi-Layer Perceptron. The objective of Multi-Layer Perception is to process the input of a node and to convert it to an output signal. While this output gets used as input in the next layer. These layers of connections help Artificial Neural Networks in learning knowledge from data sets. This structure makes sure that the Artificial Neural Network doesn't make unwanted data predictions before any prior information.

In a multi-layered network, neurons are parallelly arranged in interconnected layers. The computation unit communicates with other neurons through some type of electrical signal. Every neuron doesn't need to be activated every single time instead only the ones which are needed are activated and due to this the energy consumption is reduced. This promotes them to store data and communicate at much higher speed. This allows the usability of Neuromorphic chips in various applications. The physical connections between artificial neurons are just like organic brains which consist of biological neurons and the connection between them is called synapse.

Neuromorphic chips are characterized by the number of artificial neurons, they consist of. The neuromorphic chips launched by INTEL are Loihi and pohoiki computers.

- One loihi chip contains 1,31,000 neurons and 130 million synapses. 
- Whereas pohoiki chips contain 8.3 million neurons.

- Tianjic chip contains 40,000 artificial neurons and 10 million synapses in an area of 3.8 square millimeters.

- IBM's TrueNorth chip has 1 million neurons and over 268 million synapses. It is 10,000 times more energy-efficient than conventional microprocessors and only uses power when necessary.

- Alexnet, an image classification network has more than 62 million parameters.

- OpenAI's GPT-2 language model contains more than one billion parameters

Compared to GPUs, the tianjic chip performs 1.6-100x faster and consumes 12-10,000x less power. Also, The Pohoiki delivers 1000x better performance and is 10,000x more energy efficient than equivalent GPUs.

\section{DEEP DIVE INTO NEUROMORPHISM}

Neuromorphic scientists deeply study the functioning of human brain, the role of neurons and build a neural network based on their understanding of neurons. But there has not been any work done on the basis of synapses yet. Synapse is a part of nervous system. It is a structure that permits a neuron to pass an electrical or chemical signal to another neuron or to the target effector cell. In many synapses, the presynaptic part is located on an axon and the postsynaptic part is located on a dendrite or soma.

Synapses are the main reason our brain works; our body functions and we respond. And, the more connected neurons are the more efficient the biological system becomes at solving problems. So, when we want to take a decision the neuron fires a signal which allows other neurons to allocate themselves accordingly for the other neurons make decisions regarding the issue.So, synapses play an important role in the neural network. Therefore, the greater number of neurons in the chip, the better the functionality and results the chip can provide.

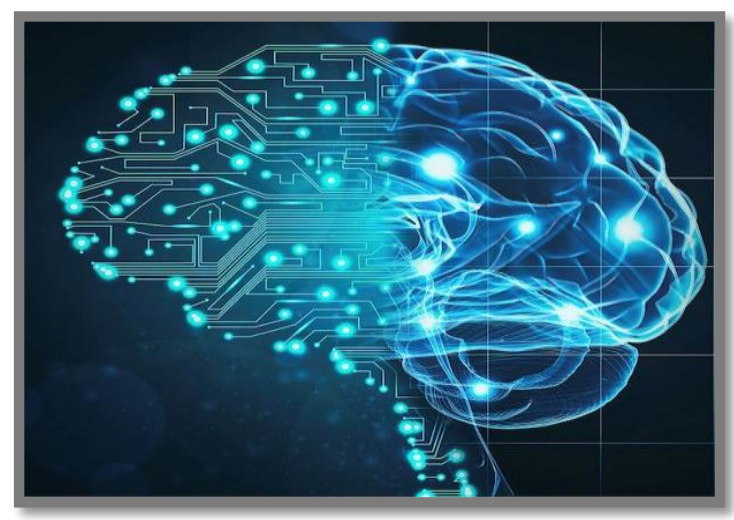

Fig.3.2 Function of Human Brain

To make synaptic connections, is one of the most significant challenges that scientists had to overcome to build neuromorphic processors. But continuous research and development in this field by MIT researchers led them to the invention of artificial synapse which works somewhat similar and gave promising results. After the invention of artificial synapse, they also built synaptic chips and were able to monitor signal strength flowing through them accurately. By testing of these chips, developers found that, in simulations, the chip and its 
synapses could be used to recognize samples of handwriting with up to $95 \%$ accuracy. Memristors can be used as super dense non-volatile memories for building instant turn on computers.

Neuroscientists after continuous research have observed learning at the level of single synapse and have gained a deep insight in how learning occurs at this scale. Psychologists have studied deeply about how a neural network work and have experimented with mammals such as rats to develop their understanding of living intelligent behaviours. Neuromorphic scientists have developed neural models to describe how a neural network works and their resulting behaviours. But they are facing many challenges as they dig deeper into the matter. One of the biggest challenges is to develop a hardware which gives following benefits:

- More computation

- Less energy consumption

- Less time consumption

- Simpler structure

- Robustness to noise

- Fault tolerance

- Physical size

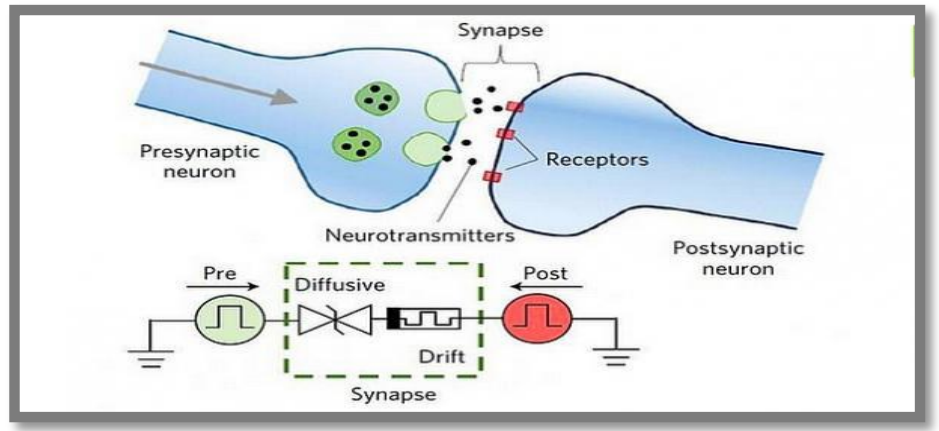

Fig. 3.3 Synapses process

These are few limitations too in the development of neuromorphism. Different types of hardware's are being designed for neural computing. Recently, the development of memeristors has increased the hope of neuromorphic scientists' community to provide low power building block to design high density memory systems for intelligent machines. These machines are designed and developed in a way keeping in mind that it not only favours scientists and narrows down to make machine intelligence but also be used and demanded by businesses to solve problem in their domains.

\section{PRESENT-DAY PROGRESS}

There are many companies and researchers experimenting on the neuromorphic models to make a working model but few of them are ahead in the race of development. The important developments made so far are: 


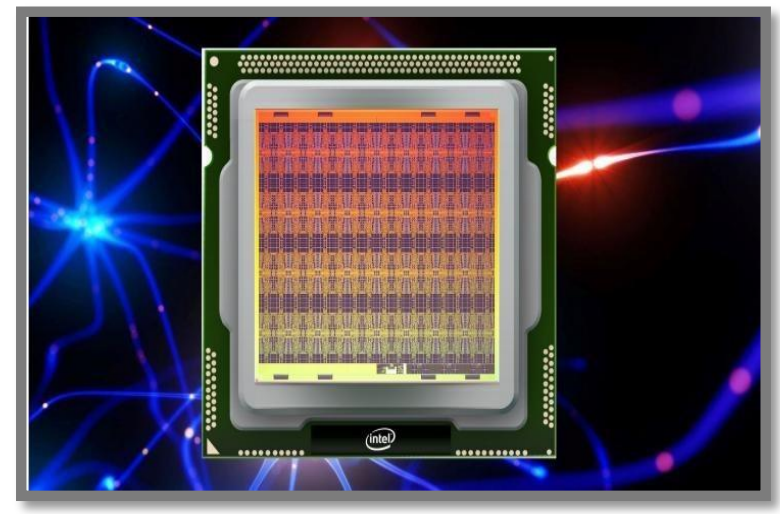

Fig.3.4 Intel's Loihi chip

Intel's Loihi: - Intel has introduces a self-learning 14-nanometer chip with over 2billion transistors, having three managing cores and having a programmable engine for on chip training of spiking neural networks. The cores have a built-in learning module in each of them and around 131,000 neurons that can convey information between one another, allowing the chip to adapt and operate based on the various conditions received from the environment. Loihi is capable of identifying 10 hazardous materials by smelling them faster than sniffer dogs. Above this it can even detect toxic fumes and diseases and can enhance its learning. It is expected to make its own decisions in the future. This energy structured chip can use date to comprehend itself and make changes. Loihi learns to be smart overtime without the need for training.

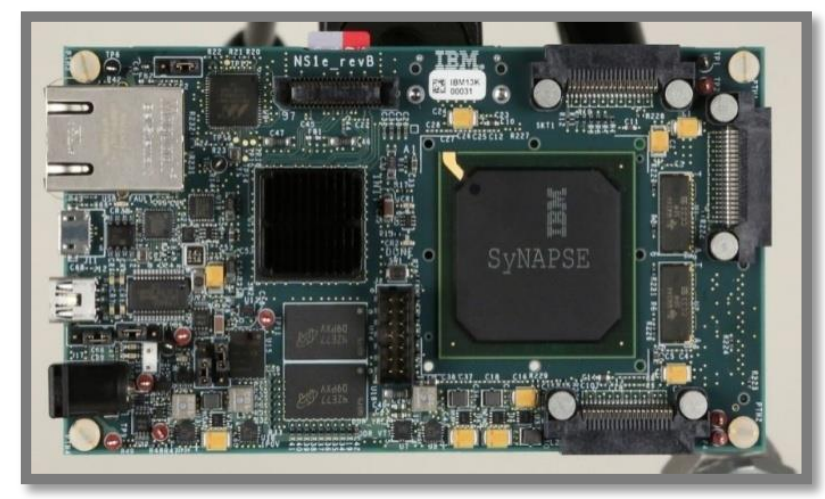

Fig.3.5 IBM's TrueNorth chip

IBM's TrueNorth: -

The abilities of TrueNorth are it can solve problems like vision, audition and multisensory fusion. It can even process the noisy sensory data efficiently. It has 4,096 cores, Samsung's $28 \mathrm{~nm}$ process with $5, .4$ billion transistors. TrueNorth is IBM's biggest chip in terms of number of transistors and it consumes less amount of power while stimulating the process. The density of TrueNorth chips power is $20 \mathrm{~W} / \mathrm{cm} 2$.It has the ability to bring a change in present computers by stimulating brain like functions inside the computer by changing the way present time concept of power and speed. It supports standard frameworks in deep 
learning which can be used during training. It's the present lead in transistors compared with other neuromorphic chips.

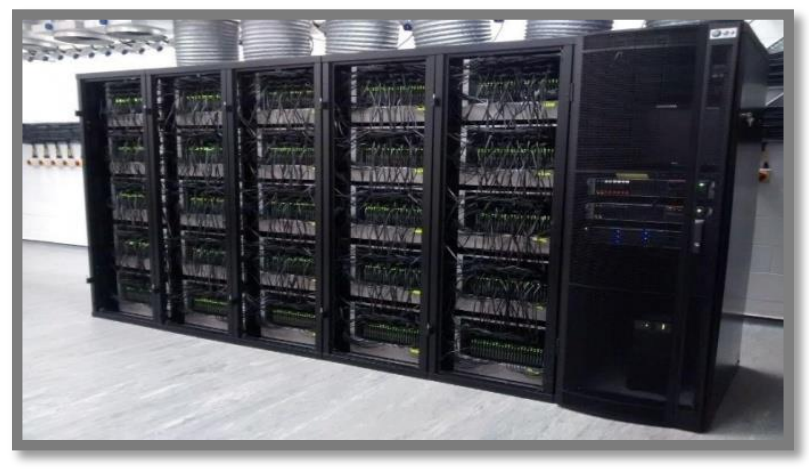

Fig.3.6 SpiNNaker supercomputer

\section{SpiNNaker:-}

Engineers of Germany's Julich Research Centers Institute of Neuroscience and Medicine, associated with United Kingdoms Advanced Processor Technologies Group at the University of Manchester. Spinnaker conducted the largest stimulation of so called largest neural network till now, which involved around 80,000 neurons connected by around 300 million synapses. Spinnaker is a low-grade supercomputer which had the ability to run such a function so-called cortical microcircuit. It simulated on a slower time scale than it was expected.

\section{MIT's: -}

A chip with more than 100 trillion synapses that act like neural network in the brain was made from silicon germanium in MIT. It showed 95 percent accuracy in human handwriting in a simulation conducted. It could also perform inference tasks like improving the detail or blurring the original image on command. This chip could help mankind in making humanoids and autonomous driving technology.

\section{Qualcomm Zeroth: -}

Qualcomm is in the process of developing a new computer structure that eliminates the old theory of computer. Working on main goals of Biologically inspired learning, making it possible for technology to reach the world has humans do and enabling the devices to view and creating and defining Neural Processing Unit (NPU), it is new technology initiated by Qualcomm which will allow devices to train for human-like interaction and responses.

\section{AkidaNSoc: -}

A brain chip has been developed by the company. Describing it as a neuromorphic engineered chip which runs on a digital logic process. Based on the biological functions so called spiral neural networks (SNNs) which consume less power than the convolutional neural networks (CNNs). The algorithmic based intensive convolutions and Propagation training methods of CNNs are replaced with neuron functions and feed forwarding training ideas. 


\section{Neromorphic Computing Analysis}

The focus of research is majorly to make developments in the field of learning from unstructured stimuli using less energy has much as possible. The building blocks in the system are analogous to neurons. Every neuron the system is capable of firing independently which then sends signals to others neurons allowing them to arrange their electric state of accordingly.

The key challenge faced by the researchers is to modulate the uncertainty and noise into natural data, which humans are able to do but the system is having trouble with. The ability to overcome this will let it to analyze a situation properly and understand the uncertainties to compute with, allowing this kind of technology in different domain applications. This kind of decision making allows them to understand and adapt to the environment which helps in predicting the further events to take a more accurate decision.

Algorithms are one of the major questions when it comes to neuromorphic computing. Since the algorithm chosen has an impact on the neuron, synapse and neural network. So, the question rises on what algorithm should be chosen. Beyond that another issue faced by the computing is whether the training of the chip should be on its own or the algorithm should be trained and then put on the chip. But since it works on learning from its surroundings the idea of chip on its own is preferred for making more better decisions in the near future by analysing each and every case.

Another issue is what happens when the algorithms are on the chip should it be monitored or shouldn't it be. So, should it learn offline and then act being monitored. One of the major reasons for neuromorphic computing to be of much interest is due to the declining of Moore's law. Even though all the perceptions are being taken most of the well-funded companies are still working hard to develop algorithms which can be used in the hardware for better efficiency of the chip. All over algorithms play a vital role in the development of neuromorphic computing.

\section{A. THRIVING OF NEUROMORPHIC COMPUTING}

The advances in transistors from a long time have reduced the size time by time, but there could be a limit to the limits extension of reducing the size where increasing the workload may not have any effect or better results. Neuromorphic computing shows a positive response in the leap towards future. The seek to make a machine on the rational theory of how the human mind may work will pay off. The attribute of neuromorphic computing is that due to its low power consumption it can be used in mobile devices and sensors in real time.

Neuromorphic computing is not a tool to make more calculations easier or create better software; the aim of it is to take decisions, responses and behaviour like a human by analysing and learning each and every scenario. In the upcoming few years, neuromorphic computing technology will become common and change the perspective we view and work with technology. The scope of Neuromorphic computing coming is promising due to its collaborative way of thinking of making computers think and work as humans. The systematic improvement in technology will thrive neuromorphic computing as a major criterion for all AI based applications and devices in the near future. 


\section{B. GOALS OF NEUROMORPHIC COMPUTING}

One of the many aspects of this research is that, while we research about the human brain and how it works and try to implement, we also get to know what it cannot do. Digitals computers are designed in a way that it can solve only particular problems and their reach is limited. If a new problem is given, it won't be able to do. On the other hand, a human being first analyses the situation and act according to its intellect. This sophistication is not developed into computers. But the problem is that a human being sometimes makes wrong decisions based on their intellect. So, the goal is to make a computer which acts and works like brain, whose structure is based on synaptic networks but in a better way.

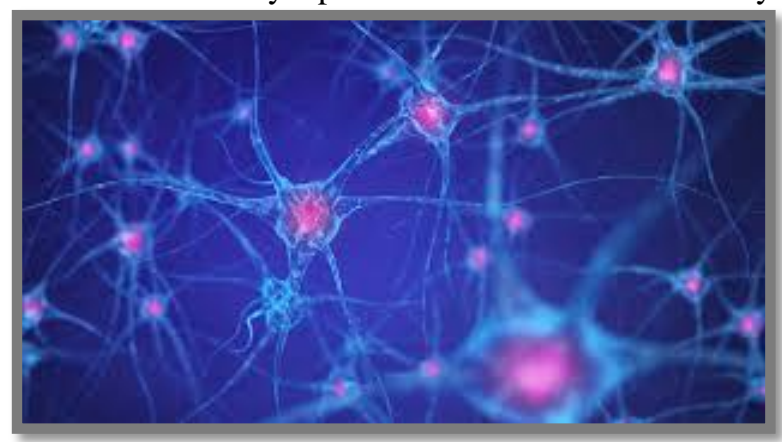

Fig.4.1 Synaptic Network

Neuromorphic computing initially came into picture in early 1980s. carver meads was verykeen to know the communication among the dense collection of wires on a transistor chip.Deep diving into the machinery, if the wires are structured more like neural networks inbrain, it could achieve its best efficiency.Many researches have been done since then by some of the big names in the industry likeIBM, HP and many more.

Human brain is efficient and there is no comparison between the efficiency of a humanbrain and digital computers. This efficiency is due to the spikes used in human brain. If aspiking artificial neuron is made, power consumption would decrease with a higher speed. This observation was made by machine learning pioneer Terry Sejnowski.

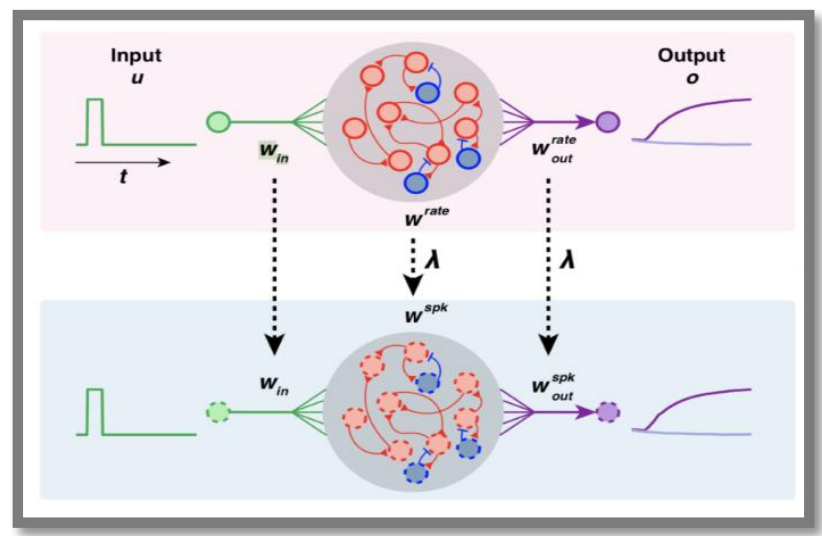

Fig.4.2 Spiking Neurons 


\section{THE HURDLES}

This is a technology which is still being researched which created a spark in advancement due to recent attempts at the practical use of neuromorphic computing architectures. The recent developments in neural networks have shown the potential to improve the efficiency in neuromorphic hardware. We are not able to see a constant growth in software field in this technology due to lack of software engineers and software developers. On the other hand, there are a handful of hardware developers, neuroscientists and researchers in the hardware sector of the field which are showing promising results.

The main challenge faced by researchers is mapping the algorithms to the architecture. It is lacking in developers and engineers whose thought should surpass our current one step at time thinking to programming. Since this field is still in the emerging stage a lot individuals do not have any particular knowledge regarding this domain, which needs to considered seriously and measures should be taken should popularize this sector. A different kind of approach is needed to access and monitor this subject. The need to combine with other fields of research such as analysis sector is needed to overcome the difficulty to manage learning continuously, accountability and the ability to handle noisy input.

The lack of perspective in programmers to develop algorithms to implement it in hardware is also another difficulty being faced by all the researchers in the recent times. The technology in which we are developing the chip isn't enough to improve the models as the capacity of better output is being limited due to the compression is size. Therefore, a new procedure is needed to implement the process.

Neuromorphic perspective has shown a significant result in identifying patterns with less amount of energy and the speed of identification can still be increased to solve numerous numbers of problems. Such a processor helps in analysing and processing data of images and videos more rapidly. Researchers need to find ways to evaluate and analyse the potentials of the available programming models and architectures to be enhanced. Regardless of all the challenges being faced, there has been enormous funding in this field.

\section{Upcoming Reach}

When Neuromorphic computing will come into use, they would be able to run algorithms (or) decisions on their own according to the necessary situation. They would be competent enough to adjust to their surroundings and will be able to differentiate between the tasks which are important and gather the information needed for the task much like a regular human brain. Rather than working on a specific set of codes. This connection between the brain and AI can help the new generation researchers in getting a better perception of the brain. The impact of neuromorphic computing can change our interface in relation to machines. Neuromorphic computing seeks to change the way machines work by letting them to analyse through monitoring.

Let's say for example, a program to recognize all kinds of animals is done by providing it with a lot of photos of all kinds of animals by a coder. A neuromorphic computer then would be able to work out the difference between all the animals and start making its own distinguished layout configurationally, which lets it to characterize much faster for the 
upcoming times. This shows that neuromorphic computing uses information given to it to understand the basic line of instructions and then, starts computing its own set of codes to make the program run quicker.

Some applications of neuromorphic computing will include the systematic understanding of irrigation between a farmer and the crop, detection of biological or chemical explosives, fraud in cheques, helping in detection of cancer, monitoring or surveillance of traffic, drones with neuromorphic technology could monitor the traffic all the time and adjust the route for traveller's time to time, Neuromorphic chips can be implanted into Robots to make autonomous decisions in fields of recruitment and warfare.

Coming to the environment point of view it could analyze the previous data, predict the exact circumstances and take measures to stop it from happening. Neuromorphic computers could bring a great knock to the stock analysis and weather forecasting. This kind of technology sees that the work gets finished faster and more effectively by always letting its network adjust and adapt to the new information. The use of this kind of technology is endless.

These are just a few capable abilities of neuromorphic computers. This technology in the upcoming days will bring an evolution to the human computers by enhancing their strengths, abilities and efficiency. Few real-world examples of neuromorphic computing are listed here:

- TianjicChip:

This chip is used in a self-driving bike which has the ability to follow a person, overcome obstacles and respond to the person's voice commands.

- Intel's Loihi chip:

This chip could recognize the smell of 10 hazardous chemicals, even chemicals such as acetone, ammonia and methane. It was able to do this even when there was a lot of noise.

- IBM's TrueNorth chip: trucks.

This chip showed that it could differentiate between pedestrians, bicyclists, cars and

The major players in neuromorphic approach are IBM, Samsung, Intel, HP, HRL, LLC, General Vision, Applied Brain Research and BrainChip Holdings.

\section{Conclusion}

Neuromorphic chips portray a solution on Nero biological concepts regarding the technical queries on computing that are being raised. Numerous applications that are being developed require enhancing large amounts of data at the initial stages and then to draw different kinds of conclusions to make a perfect decision in the environment. The expectations on the super computers which can act like a brain is to be embraced but still, as these chips are under experimental phase of development much is unexplored. Although questions are being raised on the capabilities, several researchers believe that the ability to bring a revolution to algorithms is neuromorphic computing. 


\section{References}

[1] Ankayarkanni B, Albert Mayan J, Aruna J,"Support vector machine for effective robust visual tracking", Journal of Computational and Theoretical Nanoscience,Vol.16, No.8, pp. 3571 $3575,2019$.

[2] https://bdtechtalks.com/2019/10/28/neuromorphic-chips-artificial-intelligence/

[3] https://www.zdnet.com/article/what-neuromorphic-engineering-is-and-why-its-triggered

[4] https://www.pnnl.gov/computing/HPDA/ResearchAreas/Tasks/HPDA_NeuromorphicComputi ng_17.pdf.

[5] Sathya Bama Krishna R, Usha Nandini D, Prince Mary S , 'A study on unsupervised feature selection",Journal of Advanced Research in Dynamical and Control Systems, Vol.11, No. 8 , pp. 1252-1257, 2019

[6] https://www.google.com/imgres?imgurl=https://www.intel.com/content/dam/www/public/us/e n/images/research/16x9/labs-article-neuromorphic-research-loihi-background16x9.jpg.rendition.intel.web.864.486.jpg\&amp;imgrefurl=https://www.intel.com/content/www /us/en/res.

[7] Prince Mary S, Ankayarkanni B, Usha Nandini D, Sathya Bama Krishna R, Aravindhan S,"A Survey on Image Segmentation Using Deep Learning",Journal of Physics: Conference Series, Vol.1712, No.1, 2020

[8] Albert Mayan J, Velmurugan A, Nitin Narayanan Kokkoori, Lokesh Koleti,"Forecasting Hospital Admissions in Emergency Department using Data Mining",Journal of Critical Reviews, Vol. 7, Issue.15,pp. 356-362,2020.

[9] Anto Praveena M.D. , Bharathi B,"Computation of missing fields based on neural network in the field of healthcare", International Journal of Advanced Science and Technology,Vol. 28, No. 13, pp. 366-375, 2019.

[10] Feynman Rambus. (n.d.). Retrieved from Rambus: https://www.rambus.com/blogs/lets-talkabout-neuromorphic-computing.

[11] https://en.wikipedia.org/wiki/Neuromorphic_engineering.ieved from YouTube: https://www.youtube.com/watch?v=TetLY4gPDpo.

[12] Christina R, Liz Shajan G, Ankayarkanni B,"CART- A Statistical Model for Predicting QoE using Machine Learning in Smartphones",IOP Conference Series: Materials Science and Engineering,Vol.590, No.1,2019. 